\title{
Preoperative Neutrophil to Lymphocyte Ratio and Albumin Level as Predictors for Postoperative Complication in Patients with Colorectal Cancer
}

\author{
Kyung Pil Kang, M.D., Young Hun Kim, M.D., Kyung Jong Kim, M.D. PhD. \\ Department of Surgery, Chosun University College of Medicine, Gwangju, Korea
}

\begin{abstract}
Purpose: Systematic inflammatory response biomarkers are recognized as potential prognostic factors for colorectal cancer (CRC). Recently, the neutrophil-to-lymphocyte ratio (NLR) has emerged as a possible marker for predicting the outcomes of patients with CRC. The purpose of the current study was to determine if NLR could function as a predictive marker of postoperative complications in patients with colorectal cancer who were treated surgically. Materials and Methods: One hundred and seven patients who underwent radical surgery for colorectal cancer were enrolled in the study. The NLR values were determined from the complete blood counts within one month before surgery. Values of less than or greater than 3 were defined as low (NLR-low) or high (NLR-high), respectively. Statistical comparisons were made between the NLR and the clinical-pathological variables.

Results: Sixty-eight patients met the criteria of NLR-low, and 39 patients were categorized as NLR-high. The NLR status was significantly correlated with T-stage, perineural invasion, and an increased likelihood of complications. Univariate analysis indicated that both low albumin and meeting the criteria for the NLR-high group correlated with an increased occurrence of complications $(\mathrm{P}=0.004, \mathrm{P}=0.004$, respectively). Multivariate analysis identified NLR-high and low albumin levels as independent predictors for complications $(\mathrm{P}=0.007$, odd ratio $=6.405, \mathrm{P}=0.016$, odd ratio $=9.641$, respectively)

Conclusion: The current results suggest that the preoperative NLR levels could be useful tools for predicting the occurrence of postoperative complications.
\end{abstract}

Keywords: Colorectal neoplasms, Neutrophil-to-lymphocyte ratio, Albumin, Postoperative complication, Predictor

\section{INTRODUCTION}

Similar to countries in the Western part of the world, Colorectal cancer (CRC) has become one of the most common cancers in South Korea, most likely due to shifts to a Western-style dietary pattern, a growing aging population. In 2017, the incidence of CRC accounted for $12.1 \%$ of all cancers in South Korea. Importantly, with recent advances in diagnostic methods and therapeutic approaches, the survival rate of patients diagnosed with CRC from 2012 to 2016 has increased to $75.9 \%$.[1]

Chemotherapy, radiation therapy, and surgical intervention are commonly used to treat CRC. Of these approaches, the most crucial and effective procedure is radical surgery. The most frequent complications after surgery for CRC include bleeding, peritonitis, anastomotic leakage, surgical site infection, bowel obstruction, and urinary tract infection. Additionally, as most surgical site wounds are clean-contaminated,

Received November 12, 2020; Revised November 17, 2020; Accepted November 18, 2020

Corresponding author: Kyung Jong Kim

E-mail kjkim@chosun.ac.kr@iD) ORCID https://orcid.org/0000-0001-8984-2180

This study was supported by research fund from Chosun University Hospital in 2019.

(i) \& This is an Open Access article distributed under the terms of the Creative Commons Attribution Non-Commercial License (http://creativecommons.org/licenses/ by-nc/4.0), which permits unrestricted non-commercial use, distribution, and reproduction in any medium, provided the original work is properly cited. 
they are at increased risk for infectious and inflammatory complications such as peritonitis, anastomotic leak, and surgical site infection. Importantly, peritonitis and anastomotic leak pose the greatest threat to life and impact the long-term prognosis of patients. Minimizing postoperative complications after surgery for CRC is crucial to improve postoperative outcomes, and assessing the risk for postoperative complications in advance would be advantageous for postoperative management.

Increased systemic inflammatory response influences tumor growth, progression, and prognosis. Numerous markers for a systemic inflammatory response have been identified [2] and multiple studies have investigated the wide range of markers including neutrophils, lymphocytes, platelets, C-reactive protein (CRP), albumin, Glasgow Prognostic Score (GPS), and neutrophil-lymphocyte ratio (NLR) among others. Of these, neutrophil, platelet, CRP, and albumin have been identified as oncologic prognostic factors after surgery. $[3,4]$ The NLR is calculated by dividing the absolute neutrophil count by the absolute lymphocyte count. Elevated NLR levels reflect enhanced inflammatory response, lowered immune function, and increased risk for systemic inflammatory response, subsequently the NLR has emerged as a clinically important indicator of systemic inflammation.[5] Taken together, these factors suggest NLR holds promise as a simple and effective indicator for screening patients at risk of developing complications. The aim of the current study was to investigate whether preoperative NLR could predict the development of postoperative complications in patients with CRC.

\section{MATERIALS AND METHODS}

\section{Patient selection}

The current study retrospectively reviewed 126 patients who underwent elective surgery in our hospital due to CRC between January 2015 and December 2017. Nineteen patients were excluded due to missing electronic medical record (EMR) data or clinical laboratory results, and the remaining 107 patients were included in this study. All procedures were approved by the Institutional Review Board (IRB) of the investigators' institution.

\section{Clinical variables and laboratory measurement}

We examined clinical variables including age, sex, obesity rate, length of hospital stay, complications, and tumor site. Pathologic variables assessed included tumor invasion ( $\mathrm{T}$ stage), lymph node (LN) metastasis ( $\mathrm{N}$ stage), lymphovascular invasion (LVI), and perineural invasion (PNI). Serum carcinoembryonic antigen (CEA) and albumin levels were determined as hematologic factors. Tumor location was classified into five zones: ascending colon, transverse colon, descending colon, sigmoid colon, and rectum. NLR values and blood test results were obtained from a peripheral blood smear performed within one month prior to surgery. Postoperative complications were grouped by Clavien-Dindo classification and those meeting the criteria for a score of grade III or higher were categorized as a major complication. The length of postoperative stay (POS) was calculated as the number of days from surgery to discharge from the hospital. Of the 107 patients in the study, six received neoadjuvant therapy, four received chemoradiotherapy for rectal cancer, and two received chemotherapy for metastatic ascending colon cancer. NLR was calculated by dividing the absolute neutrophil count by the absolute lymphocyte count. NLR values less than or equal to three were classified as low, where levels greater than 3 were defined as high. Complete blood count analyses were performed using the Sysmex XN-2000 and XN-3000 analyzers (Sysmex Corporation; Kobe, Japan). Elevations in CEA were defined as a level higher than $5 \mathrm{ng} / \mathrm{mL}$, while albumin level was considered low at levels below $3.0 \mathrm{~g} / \mathrm{dL}$. Of all 107 patients, no deaths occurred during the postoperative stay. All operations were performed by a single colorectal surgeon with more than 15 years of experience working with colorectal disease.

\section{Statistical analysis}

All statistical analyses were performed using SPSS Statistics 25.0 (Chicago, Illinois, USA). Chi-square tests were used to compare correlations among different clinicopathological variables between the two groups. Multivariate analysis was carried out using logistic regression. Statistical significance was set at $\mathrm{P}<0.05$.

\section{RESULTS}

\section{Demographic characteristics of patients (Table 1)}

Of all 107 patients, 66 were male and 41 were female. The mean age was 67.9 years $(66.6$ for males and 70.2 for females). Tumor type was defined as colon cancer in 68 patients and rectal cancer in the remaining 39 patients. The location of colon cancer was the ascending colon and cecum in 32 cases, the transverse colon in 8, and the descending and sigmoid colon in 28. Laparoscopic procedures were performed in a total of 65 cases and converted to laparotomy in nine. In preoperative blood tests, 36 (33.4\%) patients had elevated CEA levels and 29 (27.1\%) had low serum albumin levels. Subjects were divided into two groups by T-stage, either T1/T2 or T3/T4. The majority of patients $(75,70.1 \%)$ were stage T3/4. Of all 107 cases, 14 patients developed postoperative complications including anastomotic leak, surgical site infection and the presence of Clostridium difficile.

\section{Correlation between NLR and clinicopathological variables (Table 2 )}

A total of 68 patients met the criteria for the NLR-low group with the remaining 39 met the criteria for the NLRhigh group. T-stage, perineural invasion, and occurrence of complications were identified as factors correlated with 
Table 1. Characteristics of patients $(n=107)$

\begin{tabular}{|c|c|c|}
\hline Variable & $\mathbf{N}$ & $\%$ \\
\hline \multicolumn{3}{|l|}{ Age (year) } \\
\hline$<65$ & 43 & 40.2 \\
\hline$\geq 65$ & 64 & 59.8 \\
\hline \multicolumn{3}{|l|}{ Gender } \\
\hline Male & 66 & 61.7 \\
\hline Female & 41 & 38.3 \\
\hline \multicolumn{3}{|l|}{ BMI $\left(\mathrm{kg} / \mathrm{m}^{2}\right)^{*}$} \\
\hline Low $(<25)$ & 70 & 65.4 \\
\hline $\operatorname{High}(\geq 25)$ & 37 & 34.6 \\
\hline \multicolumn{3}{|l|}{ Tumor location } \\
\hline Ascending \& Cecum & 32 & 29.9 \\
\hline Transverse & 8 & 7.5 \\
\hline Descending & 3 & 2.8 \\
\hline Sigmoid & 25 & 23.4 \\
\hline Rectum & 39 & 36.4 \\
\hline \multicolumn{3}{|l|}{ Neoadjuvant therapy } \\
\hline No & 101 & 94.4 \\
\hline Yes & 6 & 5.6 \\
\hline \multicolumn{3}{|l|}{ T stage } \\
\hline $\mathrm{T} 1 / \mathrm{T} 2$ & 32 & 29.9 \\
\hline T3/T4 & 75 & 70.1 \\
\hline \multicolumn{3}{|l|}{$\mathrm{N}$ stage } \\
\hline No & 68 & 63.6 \\
\hline $\mathrm{N}+$ & 29 & 36.4 \\
\hline \multicolumn{3}{|c|}{ Lymphovascular invasion } \\
\hline No & 81 & 76.4 \\
\hline Yes & 25 & 23.6 \\
\hline \multicolumn{3}{|l|}{ Perineural invasion } \\
\hline No & 95 & 88.8 \\
\hline Yes & 12 & 11.2 \\
\hline \multicolumn{3}{|l|}{ Albumin (g/dL) } \\
\hline $\operatorname{High}(\geq 3)$ & 98 & 91.6 \\
\hline Low $(<3)$ & 9 & 8.4 \\
\hline \multicolumn{3}{|l|}{$\mathrm{NLR}^{\dagger}$} \\
\hline Low $(<3)$ & 68 & 63.6 \\
\hline $\operatorname{High}(\geq 3)$ & 39 & 36.4 \\
\hline \multicolumn{3}{|l|}{ Complication } \\
\hline No & 93 & 86.9 \\
\hline Yes & 14 & 13.1 \\
\hline
\end{tabular}

*BMI = body mass index; ${ }^{\dagger} \mathrm{NLR}=$ neutrophil-to-lymphocyte ratio.

NLR value. NLR levels were significantly elevated in cases that were categorized as $\mathrm{T} 3 / 4(\mathrm{P}=0.013)$, which comprised $44.0 \%$ of all patients, while $18.8 \%$ of patients met the criteria for stage T1/2. Perineural invasion correlated significantly with NLR status ( $\mathrm{P}=0.03)$, developing in $75.6 \%$ of patients in the NLR-high group and $25 \%$ of patients in the NLR-low group. There was a statistically significant correlation between NLR group and postoperative complications $(\mathrm{P}=0.04)$. Of patients with complications, $25.6 \%$ were categorized as NLR high and $4.4 \%$ were designated as NLR low. There were no significant correlations between NLR any other factors.

\section{Clinicopathological findings associated with the occurrence of complications (Tables 3,4 )}

Univariate analysis found that occurrence of complications was significantly associated with the clinicopathological variables of serum albumin and NLR. Complications occurred in $44.4 \%$ of patients in the low albumin group, while at a significantly lower rate of $10.2 \%$ of patients in the normal albumin group $(\mathrm{P}=0.04)$. The univariate analysis revealed that complications occurred in 10 of 39 patients in the NLR-high group, and 4 of 68 in the NLR-low group, which was statistically significant $(\mathrm{P}=0.04)$.

Multivariate analysis examining the association between complications and clinicopathological variables revealed that being categorized into the NLR-high group and having low albumin levels were independent predictors for the occurrence of complications $(P=0.009$, odd ratio 6.276, $P=0.016$, odd ratio 9.827).

\section{DISCUSSION}

This study investigated the ability of the NLR and albumin to significantly predict short-term postoperative outcomes after surgery for CRC. Finding indicate that the incidence of postoperative complications increased when NLR was elevated and albumin levels decreased in a group of 107 patients who underwent elective surgery to treat CRC. This outcome suggests that increased NLR and decreased albumin extended the length of hospital stay after surgery.

NLR, a value for determining systemic inflammatory and immunologic status in cancer patients, has been recognized as an indicator for predicting prognosis and recurrence in patients with CRC or other solid tumors. Gomez et al. described that disease-free survival was lowered with elevated NLR in 96 patients who received hepatectomy due to hepatocellular carcinoma, which they attributed to the fact that lymphocytes are mediators of the host's immune response against tumors.[6] The association of NLR with overall survival rate in patients who underwent surgical resection for gastric cancer has been similarly well documented.[7,8] It has been reported that increases in preoperative NLR were associated with overall survival and time to tumor progression (TTP) after surgical resection in patients with soft tissue sarcoma. [9] Notably, NLR has been used as a predictor in non-surgical patients with inflammatory disorders. For example, increased NLR in patients with ulcerative colitis has been identified as a predictor for disease severity as levels are significantly higher 
Table 2. Relationship of NLR* with various clinicopathologic parameters

\begin{tabular}{|c|c|c|c|}
\hline \multirow{2}{*}{ Variable } & Low NLR group & High NLR group & \multirow{2}{*}{ P-value } \\
\hline & NLR $<3$ & $\mathbf{N L R} \geq 3$ & \\
\hline Age (year) & & & 0.273 \\
\hline$<65$ & $30(44.1 \%)$ & $13(33.3 \%)$ & \\
\hline$\geq 65$ & $38(55.9 \%)$ & $26(66.7 \%)$ & \\
\hline Gender & & & 0.396 \\
\hline Male & $44(64.7 \%)$ & $22(56.4 \%)$ & \\
\hline Female & $24(35.3 \%)$ & $17(43.6 \%)$ & \\
\hline Location & & & 0.245 \\
\hline Colon & $46(67.6 \%)$ & $22(56.4 \%)$ & \\
\hline Rectum & $22(32.4 \%)$ & $17(43.6 \%)$ & \\
\hline Neoadjuvant therapy & & & 0.113 \\
\hline Yes & $2(2.9 \%)$ & $4(10.3 \%)$ & \\
\hline No & $66(97.1 \%)$ & $35(89.7 \%)$ & \\
\hline T stage & & & 0.013 \\
\hline $\mathrm{T} 1 / \mathrm{T} 2$ & $26(38.2 \%)$ & $6(15.4 \%)$ & \\
\hline T3/T4 & $42(61.8 \%)$ & $33(84.6 \%)$ & \\
\hline $\mathrm{N}$ stage & & & 0.456 \\
\hline No & $45(66.2 \%)$ & $23(59.0 \%)$ & \\
\hline $\mathrm{N}+$ & $23(33.8 \%)$ & $16(41.0 \%)$ & \\
\hline Lymphovascular invasion & & & 0.099 \\
\hline No & $55(80.9 \%)$ & $26(66.7 \%)$ & \\
\hline Yes & $13(19.1 \%)$ & $13(33.3 \%)$ & \\
\hline Perineural invasion & & & 0.003 \\
\hline No & $65(95.6 \%)$ & $30(76.9 \%)$ & \\
\hline Yes & $3(4.4 \%)$ & $9(23.1 \%)$ & \\
\hline Complication & & & 0.04 \\
\hline No & $64(94.1 \%)$ & $29(74.4 \%)$ & \\
\hline Yes & $4(5.9 \%)$ & $10(25.6 \%)$ & \\
\hline Albumin (g/dL) & & & 0.213 \\
\hline Low $(<3)$ & $4(5.9 \%)$ & $5(12.8 \%)$ & \\
\hline $\operatorname{High}(\geq 3)$ & $64(94.1 \%)$ & $34(87.2 \%)$ & \\
\hline $\mathrm{CEA}^{\dagger}(\mathrm{ng} / \mathrm{mL})$ & & & 0.672 \\
\hline Normal $(<5)$ & $48(70.6 \%)$ & $26(66.7 \%)$ & \\
\hline High $(5 \geq)$ & $20(29.4 \%)$ & $13(33.3 \%)$ & \\
\hline Postoperative stay (days) & & & 0.054 \\
\hline$<13$ & $55(80.9 \%)$ & $25(64.1 \%)$ & \\
\hline$\geq 13$ & $13(19.1 \%)$ & $14(35.9 \%)$ & \\
\hline
\end{tabular}

${ }^{*} \mathrm{NLR}=$ neutrophil-to-lymphocyte ratio; ${ }^{\dagger} \mathrm{CEA}=$ carcino-embryonic antigen.

in the active phase compared to the mitigation phase of the disease.[10] In addition, elevated serum NLR has been associated with the prognosis of lung cancer and gynecologic malignancies among others.[11]

Multiple studies have defined various clinical criteria to compare NLR with the postoperative prognosis of CRC. Clinical guidelines have been determined using the receiver operating characteristic (ROC) curve of NLR.[12,13] Moreover, NLR $\geq 5$ and $\geq 3$ are used to evaluate the prognosis of patients.[14,15] The clinical application of NLR cutoff values using the ROC curve is challenging due to biases in patient characteristics. Cutoff values need to be determined based on the fact that ass one of the most widely used screening biomarkers, NLR should be easily calculated and have signifi- 
Table 3. Univariate analysis for predicting complication

\begin{tabular}{|c|c|c|c|}
\hline \multirow{2}{*}{ Variable } & \multicolumn{2}{|c|}{ Complication } & \multirow{2}{*}{ P-value } \\
\hline & No & Yes & \\
\hline Age (year) & & & 0.422 \\
\hline$<65$ & $36(38.7 \%)$ & $7(50.0 \%)$ & \\
\hline$\geq 65$ & $57(61.3 \%)$ & $7(50.0 \%)$ & \\
\hline Gender & & & 0.830 \\
\hline Male & $57(61.3 \%)$ & $9(64.3 \%)$ & \\
\hline Female & $36(38.7 \%)$ & $5(35.7 \%)$ & \\
\hline $\operatorname{BMI}\left(\mathrm{kg} / \mathrm{m}^{2}\right)^{*}$ & & & 0.612 \\
\hline $\operatorname{High}(\geq 25)$ & $33(35.5 \%)$ & $4(28.6 \%)$ & \\
\hline Low $(<25)$ & $60(64.5 \%)$ & $10(71.4 \%)$ & \\
\hline Tumr location & & & 0.951 \\
\hline Colon & $59(63.4 \%)$ & $9(64.3 \%)$ & \\
\hline Rectum & $34(36.6 \%)$ & $5(35.7 \%)$ & \\
\hline Neoadjuvant therapy & & & 1.000 \\
\hline Yes & $5(5.3 \%)$ & $1(7.1 \%)$ & \\
\hline No & $88(94.7 \%)$ & $13(92.9 \%)$ & \\
\hline T stage & & & 0.459 \\
\hline $\mathrm{T} 1 / \mathrm{T} 2$ & $29(31.2 \%)$ & $3(21,4 \%)$ & \\
\hline T3/T4 & $64(68.8 \%)$ & $11(78.6 \%)$ & \\
\hline $\mathrm{N}$ stage & & & 0.261 \\
\hline No & $61(65.6 \%)$ & $7(50.0 \%)$ & \\
\hline $\mathrm{N}+$ & $32(34.4 \%)$ & $7(50.0 \%)$ & \\
\hline Lymphovascular invasion & & & 0.240 \\
\hline Yes & $19(21.3 \%)$ & $5(35.7 \%)$ & \\
\hline No & $70(78.7 \%)$ & $9(64.3 \%)$ & \\
\hline Perineural invasion & & & 0.697 \\
\hline Yes & $10(10.8 \%)$ & $2(14.3 \%)$ & \\
\hline No & $83(89.2 \%)$ & $12(85.7 \%)$ & \\
\hline Albumin (g/dL) & & & 0.004 \\
\hline $\operatorname{High}(\geq 3)$ & $88(94.6 \%)$ & $10(71.4 \%)$ & \\
\hline Low $(<3)$ & $5(5.4 \%)$ & $4(28.6 \%)$ & \\
\hline $\mathrm{NLR}^{\dagger}$ & & & 0.004 \\
\hline $\operatorname{High}(\geq 3)$ & $29(31.2 \%)$ & $10(71.4 \%)$ & \\
\hline Low $(<3)$ & $64(68.8 \%)$ & $4(28.6 \%)$ & \\
\hline
\end{tabular}

${ }^{*} \mathrm{BMI}=$ body mass index; ${ }^{\dagger} \mathrm{NLR}=$ neutrophil-to-lymphocyte ratio.

Table 4. Multivariate analysis for risk factors for complication

\begin{tabular}{lccc}
\hline Variable & P-value & Odd raito & 95\% Cl* \\
\hline Age & 0.123 & 0.342 & $0.087 \sim 1.339$ \\
$\mathrm{BMI}^{\dagger}$ & 0.265 & 0.428 & $0.096 \sim 1.906$ \\
$\mathrm{CEA}^{\ddagger}$ & 0.264 & 0.431 & $0.099 \sim 1.888$ \\
Albumin & 0.016 & 9.641 & $1.527 \sim 60.872$ \\
$\mathrm{NLR}^{\S}$ & 0.007 & 6.405 & $1.648 \sim 24.895$ \\
\hline
\end{tabular}

${ }^{*} \mathrm{Cl}=$ confidence interval; ${ }^{\dagger} \mathrm{BMI}=$ body mass index; ${ }^{\ddagger} \mathrm{CEA}=$ carcino-embryonic antigen; ${ }^{\circledR} \mathrm{NLR}=$ neutrophil-to-lymphocyte ratio. cant clinical usefulness. Since the purpose of this study was to predict the development of short-term complications in the early postoperative period, we used a cutoff value that included extended periods. Rather than use of cutoff values calculated from a complicated statistical process, the most commonly used cutoff value of NLR $\geq 3$ was applied to determine its association with various clinical variables.[16,17]

A previous study of 223 patients who received elective and emergency surgery for CRC suggested that patients with elevated serum NLR and low albumin prior to surgery were more likely to have an extended postoperative stay as a re- 
sult of postoperative complications, similar to the outcomes of the current study.[18] Several studies have reported that increased neutrophil count and decreased lymphocyte count are related to poor prognosis in all cancers. However, measures may differ due to varying time periods for assessing neutrophil and lymphocyte counts, and obtaining blood samples. Therefore, NLR more accurately represents an inflammatory reaction to tumors rather than neutrophil and lymphocyte counts. Song et al. [19] investigated the association of postoperative prognosis with NLR, lymphocyte to monocyte ratio (LMR), platelet-to-lymphocyte ratio (PLR), and prognostic nutritional ratio in 1,744 patients who underwent surgery for CRC, confirming that NLR was superior in predicting the prognosis of patients compared to other indicators.[19] A cutoff value of 3 was used in the analysis rather than the statistical cutoff using the ROC curve, which included a relatively wide range of NLR. Thus, this study was meaningful in that it identified a significant correlation between NLR and postoperative complications.

Albumin is known to be associated with the nutritional status of patients, and many studies have examined the relationship between albumin and postoperative outcomes of CRC. Lai et al. [20] reported an increase in complications related to surgical wounds, lung, urinary system, and anastomosis in 3,849 patients with preoperative hypoalbuminemia. Similarly, and Truong et al. [21] reported that preoperative hypoalbuminemia was related to an increased postoperative hospital stay, ileus, surgical site infection, enteric or enterocutaneous fistulas, deep vein thrombosis, and inflammatory bowel disease. Findings in the present study support prior reports as low levels of preoperative albumin were significantly related to postoperative complications based on univariate and multivariate analysis.

The current study is limited by the retrospective nature of the analysis, and the lack of exclusion of patients with asymptomatic inflammatory conditions, drug history or family history. The current study was also limited by a relatively small sample size. Furthermore, the dynamic changes that occur using complete blood count (CBC) could have influenced the accuracy of test results due to differing periods of blood sample collection. Thus, it should be taken into consideration that blood sample collection was carried out within one month prior to surgery, and the results may vary due to differences in timing of collection. Although this study used a cutoff value of 3 , additional studies are warranted to determine which NLR cutoff value is most appropriate. Therefore, more prospective studies with a larger sample size in the future are crucial to elucidate the prognostic value of NLR in predicting postoperative complications of patients with CRC.

\section{CONCLUSION}

This study demonstrates that preoperative NLR and albumin levels are independent predictors for the occurrence of postoperative complications after surgery for CRC. Therefore, patients having elevated NLR and decreased albumin preoperatively require careful management and monitoring after surgery.

\section{CONFLICTS OF INTEREST}

The authors of this manuscript have no conflicts of interest to disclose.

\section{ORCID}

Kyung Pil Kang, https://orcid.org/0000-0001-7350-6393

Young Hun Kim, https://orcid.org/0000-0003-4341-6623

Kyung Jong Kim, https://orcid.org/0000-0001-8984-2180

\section{REFERENCES}

1. Jung KW, Won YJ, Kong HJ, Lee ES. Cancer statistics in Korea: incidence, mortality, survival, and prevalence in 2016. Cancer Res Treat 2019;51:417-30.

2. Guthrie GJ, Roxburgh CS, Farhan-Alanie OM, Horgan PG, McMillan DC. Comparison of the prognostic value of longitudinal measurements of systemic inflammation in patients undergoing curative resection of colorectal cancer. Br J Cancer 2013;109:24-8.

3. Guthrie GJ, Charles KA, Roxburgh CS, Horgan PG, McMillan DC, Clarke SJ. The systemic inflammation-based neutrophil-lymphocyte ratio: experience in patients with cancer. Crit Rev Oncol Hematol 2013;88:218-30.

4. Ishizuka M, Nagata H, Takagi K, Iwasaki Y, Kubota K. Combination of platelet count and neutrophil to lymphocyte ratio is a useful predictor of postoperative survival in patients with colorectal cancer. Br J Cancer 2013;109:401-7.

5. Mantovani A, Allavena P, Sica A, Balkwill F. Cancer-related inflammation. Nature 2008;454:436-44.

6. Gomez D, Farid S, Malik HZ, Young AL, Toogood GJ, Lodge JP, et al. Preoperative neutrophil-to-lymphocyte ratio as a prognostic predictor after curative resection for hepatocellular carcinoma. World J Surg 2008;32:1757-62.

7. Gunaldi M, Goksu S, Erdem D, Gunduz S, Okuturlar Y, Tiken $\mathrm{E}$, et al. Prognostic impact of platelet/lymphocyte and neutrophil/lymphocyte ratios in patients with gastric cancer: a multicenter study. Int J Clin Exp Med 2015;8:593742.

8. Sagawa M, Yagawa H, Konno S, Usuda A, Maeda H, Kim $\mathrm{K}$, et al. Significance of perioperative neutrophil lymphocyte ratio (NLR) in gastric cancer and colon cancer. Gan To Kagaku Ryoho 2016;43:1243-5. Japanese.

9. Szkandera J, Absenger G, Liegl-Atzwanger B, Pichler M, Stotz M, Samonigg H, et al. Elevated preoperative neutrophil/lymphocyte ratio is associated with poor prognosis in soft-tissue sarcoma patients. Br J Cancer 2013;108:167783.

10. Celikbilek M, Dogan S, Ozbakır O, Zararsız G, Kücük H, Gürsoy $S$, et al. Neutrophil-lymphocyte ratio as a predictor of disease severity in ulcerative colitis. J Clin Lab Anal 
2013;27:72-6.

11. Zhang J, Zhang HY, Li J, Shao XY, Zhang CX. The elevated NLR, PLR and PLT may predict the prognosis of patients with colorectal cancer: a systematic review and metaanalysis. Oncotarget 2017;8:68837-46.

12. Shibutani M, Maeda K, Nagahara H, Noda E, Ohtani H, Nishiguchi Y, et al. A high preoperative neutrophil-to-lymphocyte ratio is associated with poor survival in patients with colorectal cancer. Anticancer Res 2013;33:3291-4.

13. Ozdemir Y, Akin ML, Sucullu I, Balta AZ, Yucel E. Pretreatment neutrophil/lymphocyte ratio as a prognostic aid in colorectal cancer. Asian Pac J Cancer Prev 2014;15:264750.

14. Mallappa S, Sinha A, Gupta S, Chadwick SJ. Preoperative neutrophil to lymphocyte ratio $>5$ is a prognostic factor for recurrent colorectal cancer. Colorectal Dis 2013;15:323-8.

15. Kwon HC, Kim SH, Oh SY, Lee S, Lee JH, Choi HJ, et al. Clinical significance of preoperative neutrophil-lymphocyte versus platelet-lymphocyte ratio in patients with operable colorectal cancer. Biomarkers 2012;17:216-22.

16. Casadei-Gardini A, Scarpi E, Ulivi P, Palladino MA, Accettura C, Bernardini I, et al. Prognostic role of a new inflammatory index with neutrophil-to-lymphocyte ratio and lactate dehydrogenase (CII: Colon Inflammatory Index) in patients with metastatic colorectal cancer: results from the randomized Italian trial in advanced colorectal cancer (ITACa) study. Cancer Manag Res 2019;11:4357-69.

17. Chiang SF, Hung HY, Tang R, Changchien CR, Chen JS, You $\mathrm{YT}$, et al. Can neutrophil-to-lymphocyte ratio predict the survival of colorectal cancer patients who have received curative surgery electively? Int J Colorectal Dis 2012; 27:1347-57.

18. Gohil R, Rishi M, Tan BH. Pre-operative serum albumin and neutrophil-lymphocyte ratio are associated with prolonged hospital stay following colorectal cancer surgery. $\mathrm{Br}$ J Med Med Res 2014;4:481-7.

19. Song Y, Yang Y, Gao P, Chen X, Yu D, Xu Y, et al. The preoperative neutrophil to lymphocyte ratio is a superior indicator of prognosis compared with other inflammatory biomarkers in resectable colorectal cancer. BMC Cancer 2017;17:744.

20. Lai CC, You JF, Yeh CY, Chen JS, Tang R, Wang JY, et al. Low preoperative serum albumin in colon cancer: a risk factor for poor outcome. Int J Colorectal Dis 2011;26:47381.

21. Truong A, Hanna MH, Moghadamyeghaneh Z, Stamos MJ. Implications of preoperative hypoalbuminemia in colorectal surgery. World J Gastrointest Surg 2016;8:353-62. 